\title{
A Continuum Model for Dislocation Pile-up Problems
}

\author{
Xiaohan Zhang \\ ${ }^{a}$ Department of Mechanical Engineering, Stanford University, California
}

\begin{abstract}
A 2-d dislocation pile-up model is developed to solve problems with arrays of edge dislocations on one or multiple slip planes. The model developed in this work has four unique features: 1) As a continuum mechanics model, it captures the discrete behaviors of dislocations including the region near pile-up boundaries. 2) It allows for a general distribution of dislocations and applied boundary conditions. 3) The computational complexity does not quadratically scale with increased number of dislocations. 4) The effect of anisotropy and stacking fault energy can be naturally modeled. Pile-ups against a lock under shear load are extensively investigated, which shows the dependence of near-lock piles distribution on the total number of dislocations. The stacking fault energy effect is found to be positively correlated to the length of an equilibrated pileup. The stress intensity near a bi-metallic interface is studied for both isotropic material and anisotropic materials. The model is validated by reproducing the solutions of problems for which analytical solutions are available. More complicated phenomena such as interlacing and randomly distributed dislocations are also simulated.
\end{abstract}

Keywords: dislocation pile-ups, bi-metallic interface, anisotropy, stacking fault energy

\section{Introduction}

The prediction of plastic deformation of metals has been an important research topic for decades, which primarily reduces to the question of properly understanding the motions of dislocations as the major cause of plastic flows in metals. A modern plasticity theory, Field Dislocation Mechanics (FDM), has been devel5 oped to predict the time-dependent mechanical response of bodies containing a distribution of dislocations mathematically represented by the dislocation density tensor. FDM has been completed, generalized, and understood as a rigorous, continuum thermomechanical model of dislocation dynamics and its collective behaviors (Acharya (2001, 2003, 2004); Acharya and Roy (2006); Acharya (2010, 2011)). The theory has been majorly applied to modeling some physically interesting phenomenological plasticity problems, for instance, size effects and back-stress development (Roy and Acharya (2005); Puri et al. (2010, 2011a,b)). Although these works take into account the dislocation generation and motion statistically based on the dislocation density tensor, the discrete nature of dislocation evolution, which becomes very important when the characteristic length of internal deformation fields or external sample size is at micron/submicron scale

\footnotetext{
${ }^{*}$ Corresponding author. E-mail address: xzhang11@stanford.edu (Xiaohan Zhang).
} 
(Berdichevsky and Dimiduk (2005)), has not been fully captured. Therefore, one of the objectives of this work is to demonstrate the capability of FDM in modeling and predicting the motions of dislocation microstructures. A well-known benchmark problem that serves such a purpose is the study of dislocation pile-ups, which, in addition to providing a key mechanism for size effects, (Mesarovic et al. (2015)), also plays an important role in other phenomena such as work-hardening, yielding, and cleavage.

The solution to the dislocation pile-up problem was first attempted by Eshelby, Frank and Nabarro Eshelby et al. (1951), who framed the question as follows: considering an array of identical straight dislocations on the same slip plane forced against an impenetrable wall, what are the equilibrated positions/distribution of the dislocations and their corresponding stress fields? It should be clearly noted that the problem stated as such is mathematically simplified by the assumption of long, straight slip bands despite the fact that such slip bands are rarely observed experimentally. The impenetrable walls are also mathematically abstracted from various physical objects in general, such as grain boundaries and bimetallic interfaces (Pacheco and Mura (1969)). However, despite such simplifications, solving pile-up problems is difficult in the sense that the equilibrium state of each dislocation is determined by the combination of mutually repulsive/attractive dislocation interactions and the externally applied loads. From a numerical point of view, the computational cost of handling interactions between pairs of dislocations scales quadratically with the number of dislocations, which is known as the major bottleneck for Discrete Dislocation Methodologies in simulating strain hardening. Our model does not have this constraint but requires a good quality of mesh refinement near dislocation cores. So the computational cost scales with the number of grid points.

As a brief review, we list some classical methods that have been developed specifically for solving dislocation pile-up problems.

i The first model developed by Eshelby et al. (1951) is based on the balance of Peach Kohler forces on each dislocation, i.e.,

$$
\sum_{i=1, i \neq j}^{n} \frac{A}{x_{j}-x_{i}}+P\left(x_{j}\right)=0, j=1,2, \ldots . n .
$$

where $x_{i, j}$ are the $i, j$ th dislocation positions to be solved; $n$ is the total number of dislocations; $P\left(x_{j}\right)$ is the applied stress at the $j t h$ dislocation; $A$ is a constant depending on the dislocation type with $A=\mu b / 2 \pi(1-\nu)$ for edge dislocations. Eq. (1) is solved by introducing a polynomial,

$$
f(x)=\prod_{i=1}^{n}\left(x-x_{i}\right)
$$

such that one can equivalently convert it to the following ordinary differential equation,

$$
f^{\prime \prime}(x)+2 P(x) f^{\prime}(x)+q(n, x) f(x)=0
$$

Eq. (3) is solvable provided that $q(n, x)$ is chosen in a way such that the equation has a $n^{\text {th }}$ degree polynomial solution whose roots are real and distinct; thus $x_{j}$ can be determined as the roots of a set of orthogonal polynomials subjected to certain constraints. However, this immediately sets a 
limitation to the method since finding proper $q(n, x)$ becomes mathematically difficult for arbitrary loading/boundary conditions.

ii Head (1959) considered solving double pile-ups and interlacing problems by numerically exploring Eshelby's method. Here double pile-ups refers to a group of positive dislocations next to a negative group while both groups are forced to glide in opposite directions and form pile-ups on two sides. Interlacing refers to two groups of dislocations with opposite signs that lie on adjacent slip planes. The leading dislocations of adjacent planes may interlace and set the whole system equilibrium. Head's method requires applied stresses large enough so that dislocations cannot annihilate in double pile-ups. Also, the interlacing of more than three pairs of dislocations is reported to be intractable.

iii Leibfried $(1951,1954)$ shows that one can obtain approximate solutions by treating discrete dislocations with continuously distributed dislocation density that can be determined from an integral transform. This methodology is applied in recent works, e.g., Akarapu and Hirth (2013) and Ockendon et al. (2007), to study pile-ups and double pile-ups. However, it has been demonstrated that neglecting short range interaction effects leads to inaccurate results Roy et al. (2008). A semi-continuum version has to be developed Hall (2011) in an attempt to improve the appropriateness of Leibfried's model in approximating discrete microstructure near the pile-up head with continuous functions.

iv Voskoboinikov et al. (2007a) proposes a methodology that accommodates the near-lock behavior of pile-ups by discretely representing dislocations in the near-lock field and matching the discrete stress field with the far field stress (where dislocations are still continuously represented with dislocation density). The method is applied in solving pile-ups against bimetallic interface Voskoboinikov et al. (2007b) and Voskoboinikov et al. (2009). However, the method is only valid for constant shear load and infinite isotropic domain. Some upscaling methods for dynamics of dislocation walls by means of $\Gamma$ convergence on the space of probability measures have been recently developed (van Meurs and Muntean (2014) Scardia et al. (2014)). Those methods only deal with dislocation walls (pile-ups on $n$ slip planes with $n \rightarrow \infty$ ) despite the non-physical assumption that dislocations move in the form of walls. Rezaei Mianroodi et al. (2016) applied Peierls-Nabarro model to solve such problems and report qualitative agreement with discreteness based methods.

The methodology proposed in this work stems from the 2-d FDM framework developed in Acharya and Zhang (2014); Zhang et al. (2015), where the motion of dislocations is governed by a kinematical rule of the plastic strain implying the conservation law of the Nye tensor (The detailed proof of this geometrical argument can be traced back to Acharya (2011)). The whole system is governed by a set of partial differential equations and therefore the computational cost does not explicitly depend on the number of dislocations. This differentiates our model from all discrete-based methodologies. In the 2-d FDM approach, modeling dislocation microstructures is made possible by building multi-well non-convex inelastic energy and dislocation core energy into the system dissipation, which has the advantage in terms of capturing discreteness behaviors, compared to the continuum and semi-continuum approaches derived from Leibfried's model. This 
inelastic energy can be analogous to the stacking fault energy accounted for in phase-field (Shen and Wang (2003), Wang et al. (2001), Wang and Li (2010)) and Peierls-Nabarro (PN)-based (Hu et al. (2004), Xiang et al. (2008), Mianroodi et al. (2016)) dislocation models. We should point out that those continuum models also have potential to model equilibrated discrete dislocations and their interactions. However, none of the models have yet solved the problems presented in this paper, i.e., explicitly modeling discrete dislocations (up to 500) with nonlocal stress field in the 2-d domain.

It should be pointed out that both stacking fault energy and dislocation core energy are physical quantities and that can be fitted from finer scale calculations. Therefore, our model serves as a multiscale modeling tool that connects the mesoscale phenomena of dislocation pile-ups to the finer scale of atomic information.

In this paper, we validate our model by first solving a few classical problems for which analytical solutions

90

\section{2-D edge dislocation model derived from FDM}

We refer to Acharya and Zhang (2014) for a detailed description of the 2-d edge dislocation model, including the derivation and the numerical scheme. Here we summarize the governing equations and involved 
geometric and constitutive ingredients. Consider the following geometry as illustrated in Fig. 1:

$$
\begin{aligned}
& \Omega=\{(x, y):(x, y) \in[-W,+W] \times[-H,+H]\}, \\
& L=\{(x, y):(x, y) \in[-W,+W] \times[-b,+b]\}, \\
& 0<b<H, \quad W>0,
\end{aligned}
$$

where $b$ is the length of Burgers vector, acting as a characteristic length scale. The model may be viewed as a composite comprising layers whose response is elastic-plastic due to dislocation arrays, and elastic regions, $\Omega \backslash L$, whose stress response is purely linear elastic. The thickness of the plastic layer, i.e., the separation between the adjacent atomic planes, is assumed to be $2 b$.

The dissipation of the whole body is defined as the difference in the rate of working of external forces and the rate of stored energy and only arises from the layers (since everywhere else the body is elastic). Assuming a stored energy density function of the form

$$
\psi\left(\boldsymbol{\epsilon}^{e}, \boldsymbol{\alpha}\right)+\eta\left(\boldsymbol{U}^{p}\right)
$$

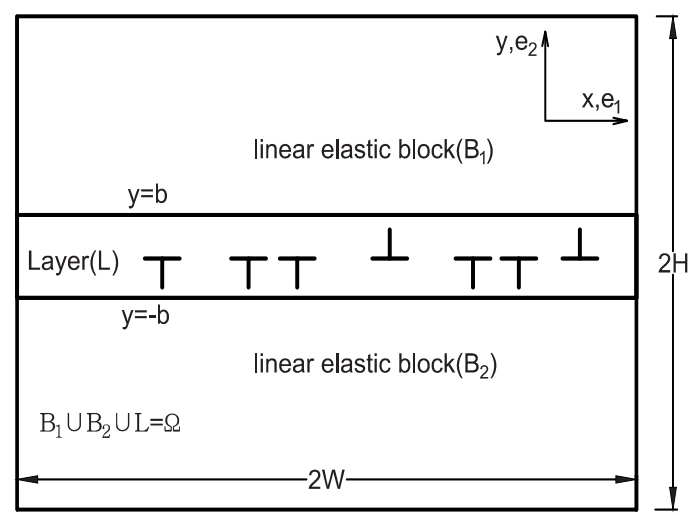

Figure 1: Schematic of a discrete edge dislocation array.

where $\boldsymbol{\epsilon}^{e}$ is the symmetric part of the elastic distortion $\boldsymbol{U}^{e}$, thus the Cauchy stress tensor is defined as $\boldsymbol{T}=\partial \psi / \partial \boldsymbol{\epsilon}^{e}$. The function $\psi$ is assumed to be positive-definite quadratic in $\boldsymbol{\epsilon}^{e}$ and the function $\eta$ is multiwell non-convex, endowing the energy function with slip barriers and conferring preferred energetic status to certain plastic strains. Together, these two functions enable the robust modeling of overall total strain distributions in the layer displaying localized, smooth transitions between slipped and unslipped regions (or between the preferred strain states encoded in $\eta$ ). This crucially requires adding an energetic penalty to the development of high values of the dislocation density $\boldsymbol{\alpha}$, referred to as a core energy. It is numerically demonstrated in Zhang et al. (2015) that the balance between these thermodynamic forces sets the dislocation 
core width at equilibrium. It is to be noted that the core energy is a fundamental physical ingredient of our model and not simply a mathematical regularization.

The following assumptions are considered for the derivation of the model:

1. small deformation;

2. plastic strain $\boldsymbol{U}^{p}$ only has one non-vanished component, defined as $\phi(x, t)$, i.e., $\phi=U_{12}^{p}$;

3. all dislocations are of edge type and move along the $\boldsymbol{e}_{1}$ direction.

The rest of the derivation follows rigorous considerations of the 2 nd thermodynamic law in the sense that the dislocation velocity function is chosen in the direction of the driving force (in the context of crystal plasticity theory Rice (1971)) to guarantee positive dissipation rate of the system at any instant. As a result, the governing equations of the derived model read,

$$
\left\{\begin{array}{l}
\rho \ddot{u}=\frac{\partial T_{i j}}{\partial x_{j}} \\
\phi_{t}=\frac{1}{B_{m} b^{m-1}}\left|\frac{\partial \phi}{\partial x_{1}}\right|^{2-m}\left(\tau-\tau^{b}+\epsilon \frac{\partial^{2} \phi}{\partial x_{1}^{2}}\right)
\end{array}\right.
$$

where

$$
\begin{aligned}
& \tau=\frac{1}{2 b} \int_{-b}^{b} T_{12}(x, y, t) d y, T_{i j}=C_{i j k l}\left(u_{k, l}-U_{k l}^{p}\right) \\
& C_{i j k l}=\lambda \delta_{i j} \delta_{k l}+\mu\left(\delta_{i k} \delta_{j l}+\delta_{i l} \delta_{j k}\right) \\
& \tau^{b}=\frac{\partial \eta}{\partial \phi}=\frac{2 \mu \bar{\phi}}{\pi} \sin \left(2 \pi \frac{\phi}{\bar{\phi}}\right) .
\end{aligned}
$$

Note that $\tau$ is defined as the shear stress averaged across a plastic layer. The material properties are exclusively controlled by Lamé constants $\lambda$, $\mu$, the drag coefficient $\tilde{B}_{m}$, the shear wave speed $V_{s}$ ( calculated as $V_{s}=\sqrt{\mu / \rho}$, ) and the diffusion parameter $\epsilon$. The exponent $m$ relates to the choice of the drag function for the dislocation velocity and is set to $m=1$ for all simulations. The system is then scaled by introducing dimensionless variables,

$$
\tilde{x}=\frac{x}{b}, \tilde{t}=\frac{V_{s} t}{b}, \tilde{u}=\frac{u}{b}, \tilde{T}=\frac{T}{\mu}, \tilde{\tau^{b}}=\frac{\tau^{b}}{\mu}, \tilde{\epsilon}=\frac{\epsilon}{\mu b^{2}}, \tilde{B}_{m}=\frac{V_{s}}{\mu / B_{m}}
$$

which leads to the following nondimensionalized form of system (5),

$$
\left\{\begin{array}{l}
\frac{\partial^{2} \tilde{u}}{\partial \tilde{t}^{2}}=\frac{\partial \tilde{T}_{i j}}{\partial \tilde{x}_{j}} \\
\frac{\partial \phi}{\partial \tilde{t}}=\frac{1}{\tilde{B}_{m}}\left|\frac{\partial \phi}{\partial \tilde{x}_{1}}\right|^{2-m}\left(\tilde{\tau}-\tilde{\tau}^{b}+\tilde{\epsilon} \frac{\partial^{2} \phi}{\partial{\tilde{x_{1}}}^{2}}\right)
\end{array}\right.
$$

In the limit of the loading rate $\Gamma$ going to zero, the time evolutionary system (7) converges to a quasi-static response. This is realized by introducing a slow time scale

$$
s=\Gamma \tilde{t}
$$


and substituting $s$ into system (7). $\Gamma \ll 1$ gives,

$$
\left\{\begin{array}{l}
0=\frac{\partial \tilde{T}_{i j}}{\partial \tilde{x}_{j}} \quad \text { in } \Omega \\
0=\frac{1}{\tilde{B}_{m}}\left|\frac{\partial \phi}{\partial \tilde{x}_{1}}\right|^{2-m}\left(\tilde{\tau}-\tilde{\tau^{b}}+\tilde{\epsilon} \frac{\partial^{2} \phi}{\partial{\tilde{x_{1}}}^{2}}\right) \quad \text { in } L
\end{array}\right.
$$

FEM is used to solve the equation for balance of linear momentum in a staggered scheme that utilizes the plastic distortion $\boldsymbol{U}^{p}$ as a given quantity obtained by evolving $\boldsymbol{U}^{p}$ ( or $\phi$ ) in the remaining part of the scheme. $\phi$ is solved from in a gradient flow fashion on slow time scale $s$ until $\left\|\phi_{s}\right\|_{\infty}$ (considered as the residual) becomes smaller than a predifined dimensionless tolerance. Namely, we evolve $\phi$ from some initial conditions $\phi(x, 0)$ according to

$$
\left\{\begin{array}{l}
0=\frac{\partial \tilde{T}_{i j}}{\partial \tilde{x}_{j}} \\
\frac{\partial \phi}{\partial s}=\frac{1}{\tilde{B}_{m}}\left|\frac{\partial \phi}{\partial x_{1}}\right|^{2-m}\left(\tau-\tau^{b}+\epsilon \frac{\partial^{2} \phi}{\partial x_{1}^{2}}\right)
\end{array}\right.
$$

until the dimensionless norm $\left\|\phi_{s}\right\|_{\infty} \leq 10^{-6}$, with balance of linear momentum satisfied at all instants. It bears an emphasis that the equilibria of $(\phi, \boldsymbol{u})$ obtained as such are not strict, as the determination of true equilibria usually requires the residual reaches below $\sim 10^{-10}$. However, as numerically demonstrated in Das et al. (2012), the translational motions of dislocations and the change of stress fields both become indistinguishable when $\left\|\phi_{s}\right\|_{\infty} \leq 5 \times 10^{-5}$. And since dislocation positions and stresses are the only two unknowns of interest for our pile-up problems, a tolerance of $10^{-6}$ serves as a conservative choice. To be complete about this statement, one might distinguish a minor change in dislocation core shape from $\left\|\phi_{s}\right\|_{\infty}=10^{-6}$ to $10^{-10}$. This core change has an approximate $10^{-5} \mu$ effect in determining Peierls stress problem (Zhang et al. (2015)).

\section{Numerical solutions to some pile-up problems}

\subsection{Dislocations lying between two locked ends.}

Consider an array of $n$ dislocations with the outer two locked. A shear load is applied through the Neumann boundary condition:

$$
\boldsymbol{t}=\tau^{a}\left(n_{2} \boldsymbol{e}_{1}+n_{1} \boldsymbol{e}_{2}\right)
$$

where $\tau^{a}$ denotes the shear load magnitude.

Recall that the spatial derivative of the plastic strain represents the dislocation density, i.e., $-\phi_{x}(:=$ $\partial \phi / \partial x)=\alpha_{13}(x)$. Therefore, one can define the initial distribution of dislocations by making use of a linear combination of the hyperbolic tangent function

$$
\phi^{q}(x)=\frac{\bar{\phi}}{2} \tanh \left(a\left(x-x_{q}\right)\right)+\frac{\bar{\phi}}{2},
$$


where $\bar{\phi}$ is chosen to be the spacing between two neighbouring preferred plastic strain states encoded in (4). $\phi_{x}^{q}$ gives a localized dislocation peak at $x_{0}$. A superposition of $\phi^{q}(x, 0)$ gives any distribution of dislocations along the slip plane at locations $x_{q}$ (the location of a dislocation is defined to be the coordinate of the peak of $\phi_{x}$ ), whose core width at equilibrium is determined by the combining effects of dislocation density gradient $\epsilon \phi_{x x}$ and the multi-well energy $\eta$. Table 1 lists all material parameters used in the simulation.

\begin{tabular}{|c|l|l|l|}
\hline Name & Physical definition & value & units \\
\hline$E$ & Young's modulus & 70 & $G P a$ \\
$\mu$ & shear modulus & 26 & $G P a$ \\
$V_{s}$ & shear wave velocity & 3130 & $\mathrm{~m} / \mathrm{s}$ \\
$\tilde{B}_{m}$ & drag coefficient & 0.0297 & $\mu / V_{s}$ \\
$\epsilon$ & core-energy strength & 0.25 & $\mu b^{2}$ \\
$W, H$ & domain width \& height & $10^{3} \times 10^{3}$ & $b \times b$ \\
$h$ & layer element width & 0.1 & $b$ \\
$\bar{\phi}$ & well spacing & 0.5 & $/$ \\
$|e|_{\phi}$ & tolerance for $\phi$ equation & $10^{-6}$ & $\mu / \tilde{B}_{m} b$ \\
\hline
\end{tabular}

Table 1: Simulation parameters.

The parameter values are not exactly for one specific material. The modulus and Poisson ratio are in accordance with Aluminum. The core energy strength has the unit of $\mu b^{2}$ whose dimensionless value is adopted from Acharya (2010) and adjusted accordingly. The drag coefficient is taken from Kubin et al. (1992). The FE mesh can largely affect the overall computational complexity. Fig. 2 shows the FE mesh used where only elements within and near the layer are highly refined by using a transition-layer meshing technique. The mesh dependence study of the model performed in Zhang et al. (2015) has demonstrated a good convergence, especially when the mesh size in the layer is below $b / 3$. All the problems presented in this paper are solved on a mesh which has layer elements of size $0.1 b$. As the first examples, we generate 5 and 7 dislocations with initial positions $(s=0)$ shown in the first row of Fig. 4(a) and 4(b). 


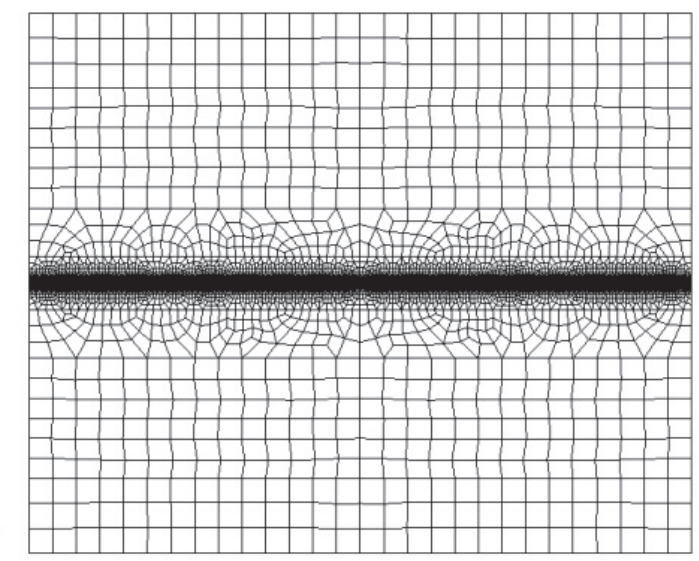

Figure 2: Transition-layer technology is used to generate a layer-refined mesh.

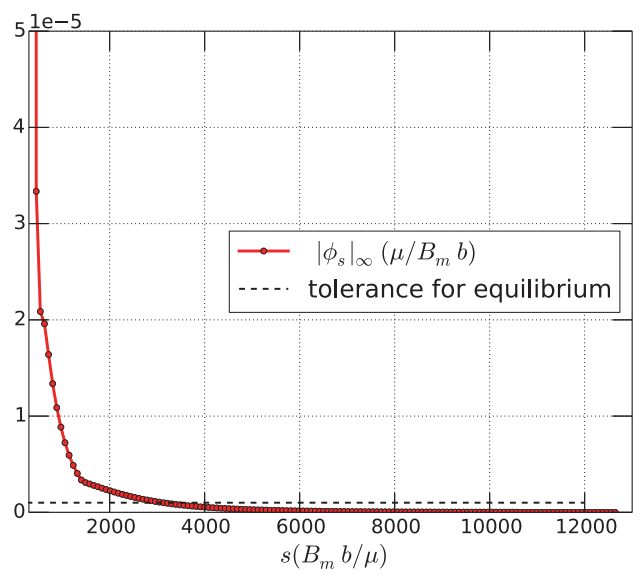

Figure 3: $\left\|\phi_{s}\right\|_{\infty}$ evolution on dimensionless quasi-static time scale $s$. The dotted line denotes the predefined tolerance for equilibrium.

We consider the loading free case which can be solved analytically (Eshelby et al. (1951)). The analytical positions are found by calculating the roots of the first derivative of the $(n-1)_{\text {th }}$ Legendre polynomial. To solve this problem with our approach, system (10) is evolved until equilibrium is numerically achieved, i.e., $\left\|\phi_{s}\right\|_{\infty}<|e|_{\phi}$, where $\left\|\phi_{s}\right\|_{\infty}$ is the $L^{\infty}$ norm of $\left|\phi_{s}\right|$. The outer two dislocations are locked by enforcing $\left\|\phi_{s}\right\|_{\infty}=0$ within their cores. During the evolution, it is observed that $\left\|\phi_{s}\right\|_{\infty}$ asymptotically goes to zero (Fig. 3) and the dislocations move towards their equilibrated positions as shown in Fig. 4(a) and 4(b). Our numerical results are found to quantitatively agree with the analytical solutions which are marked by dotted lines.
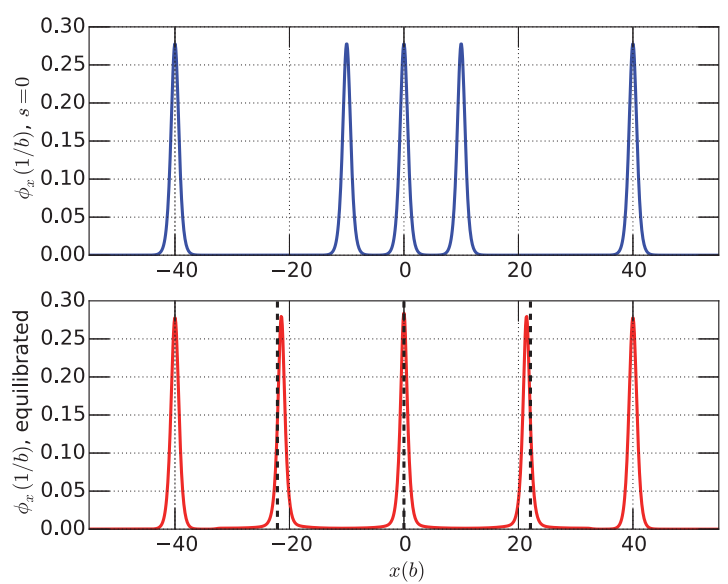

(a) $n=5$ dislocations
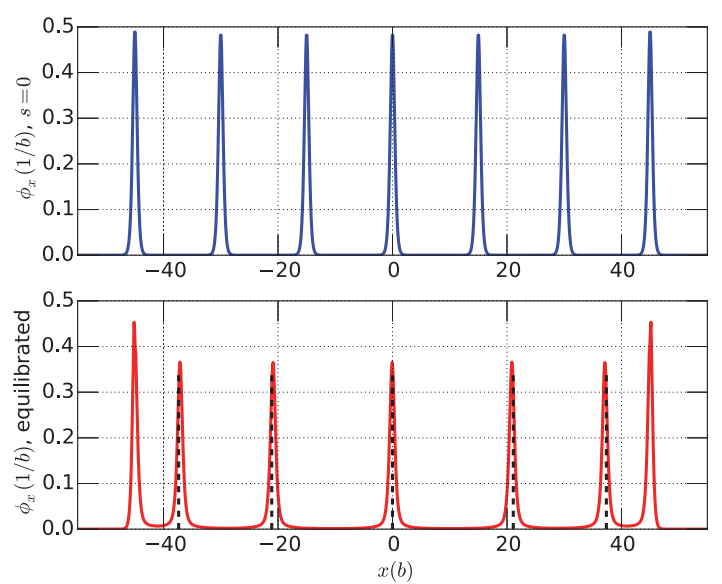

(b) $n=7$ dislocations

Figure 4: Under zero applied stress, positions of dislocation array (with outer two locked) predicted by the model agree with the analytical solution proposed by Eshelby et al. (1951) (marked by dotted lines). Top: initial condition of pile up. Bottom: equilibrated dislocation array. 


\subsection{A group of free dislocations in a transversely loaded beam}

Consider $n$ free dislocations lining up along the neutral plane of a transversely loaded beam supported at double ends. This problem was originally proposed and solved in Eshelby et al. (1951) and consequently studied in Chakravarthy and Curtin (2010) and Akarapu and Hirth (2013). Those works needed to assume a load of linear gradient form, i.e., $x(d P / d x)$ due to the 1-d set up of their models. Here we can model the loaded beam directly in 2-d, which allows for a more realistic representation of the stress field. Consider the geometry of the domain as

$$
\Omega=\{(x, y):(x, y) \in[-500 b,+500 b] \times[-25 b,+25 b]\}
$$

A transverse pressure is applied on the $y=25 b$ surface. The $n$ free dislocations are initially distributed arbitrarily along the neutral plane. One cannot analytically obtain the locations of each dislocation, but only a upper and lower bound for the final distributions. According to Eshelby et al. (1951), the equilibrated locations of dislocations are expected to lie in the region of

$$
|x|<\sqrt{\frac{(2 n+1) A}{d P / d x}}
$$

which is marked by the black dotted lines in Fig. 5(a) and 5(b). Numerical results from our model is shown in Fig. 5(a). Even when initial distributions of dislocations are far away on the right end, all dislocations move to the left with both core widths and dislocation spacings constantly being compressed during the evolution of the system. The territory of the final dislocation clusters falls exactly in the upper and lower bounds of Eq. (14). The corresponding shear stress field $\tau$ on the slip plane is shown in Fig. 5(b).
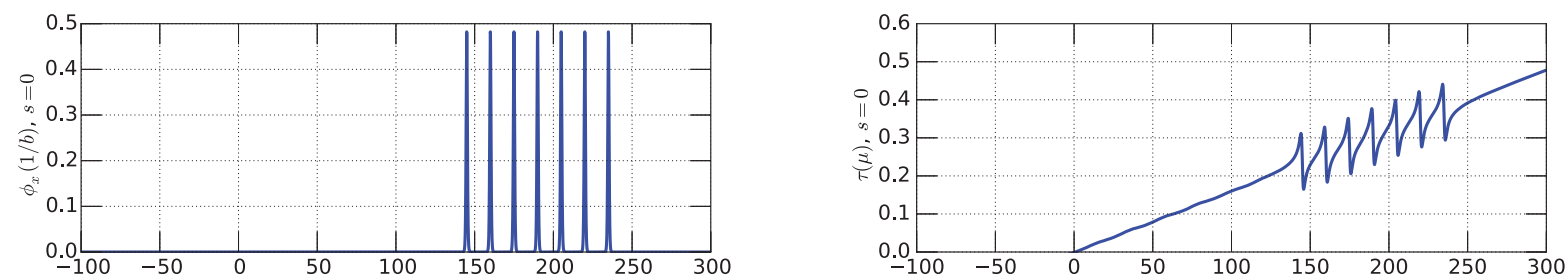

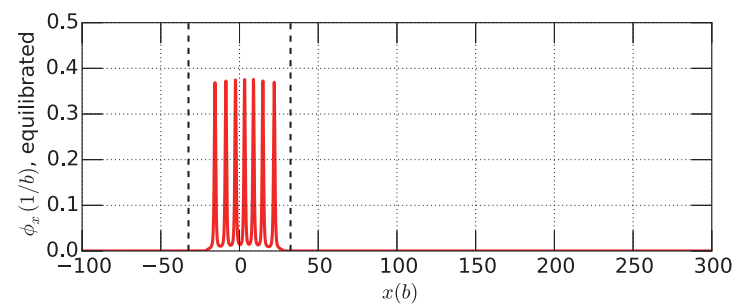

(a) Dislocation evolution.

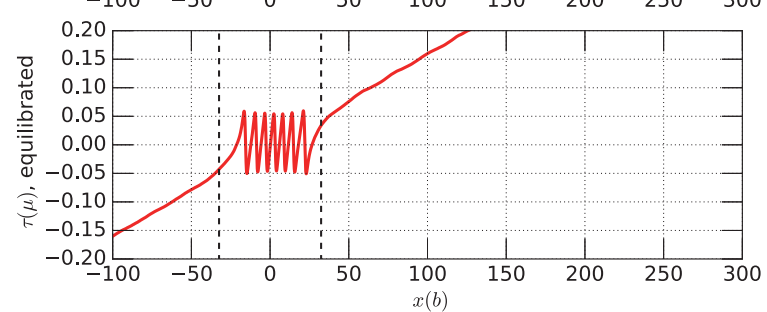

(b) Layer stress $\tau$.

Figure 5: Dislocations redistribution at equilibrium in the middle of a transversely loaded beam. Vertical lines denote the analytically predicted bounds that all dislocations fall in at equilibrium.

This example reveals the fact that dislocations can be found in the low stress region (middle of the beam) 
of a body while leaving the high stress region (boundaries) dislocation-free, which is in contradiction to what is commonly believed.

210

\subsection{A group of dislocations piled-up against a lock under constant shear load}

We first consider a set of $n=7$ dislocations forced against a leading locked dislocation at $x=0$ under shear load $\tau^{a}=0.015 \mu$. The same parameters of Table 1 are used. We are interested in comparing our results to the analytical inequalities obtained from Eshelby's model, i.e.,

1. L, the length of the slip plane occupied by dislocations, is bounded by

$$
L \leq 2 n A / \tau^{a}=113.6 b
$$

2. $\mathrm{d}$, the distance between the locked and nearest free dislocation, is bounded by

$$
d \leq 1.84 A / n \tau^{a}=2.15 b
$$

As a comparison to the discrete-based solutions above, our method yields $L=103.1 b$ and $d=2.1 b$, both consistent with the analytical evaluations. This demonstrate that the short range effect near the pile-up head is well captured by our model, as can be seen from the inset of Fig. 6(a). By fitting the shear stress field in the layer, it is also shown that the stress diverges as $1 / x$ away from the pile-up head (blue line in Fig. 6(b)):

$$
\tau^{*}(x)=\frac{-0.4887}{x-4.1345}+\tau^{a}, x \leq 0 .
$$

which suggests that the pile-up of equilibrium can be represented as a super-dislocation of Burgers vector $0.4887 / A=4.29 b$ centered at $x=4.1345 b$.
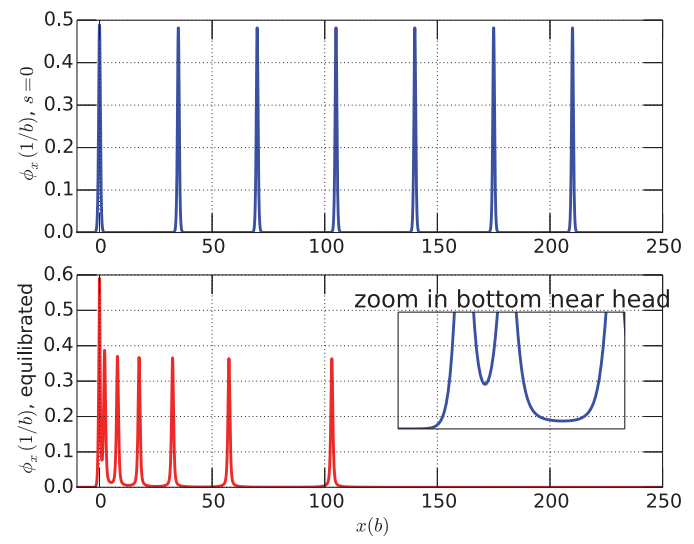

(a) Dislocations are forced to pile up. Microstructure details near the pile-up head at bottom is shown by inset plot.

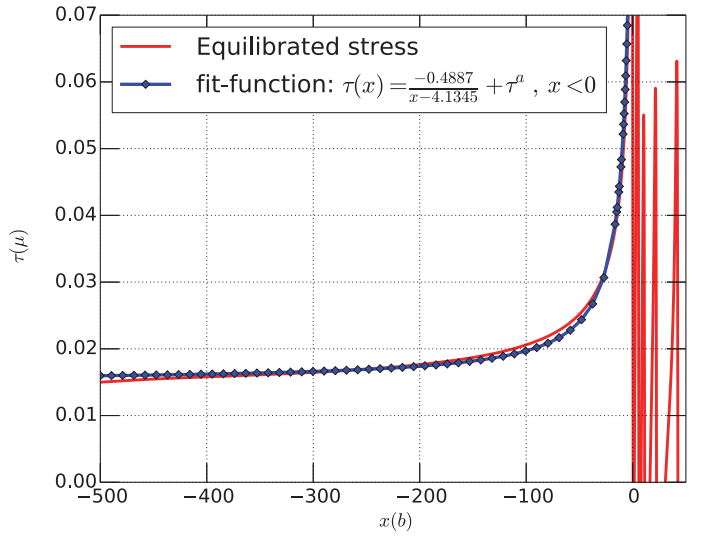

(b) fit diverging shear stress in the layer with an inverse function of the distance from the pile-up head.

Figure 6: Dislocation pile-ups under constant shear loads. Stress concentration in front of the pile-up head. 
Now we gradually increase the number of dislocations in the pile-up, from 30 to 100, as shown in Fig. 7. We compare our solutions to that obtained from Voskoboinikov et al. (2007a). It is found that the agreement density distribution near the pile-up boundary compared to discreteness-based models.

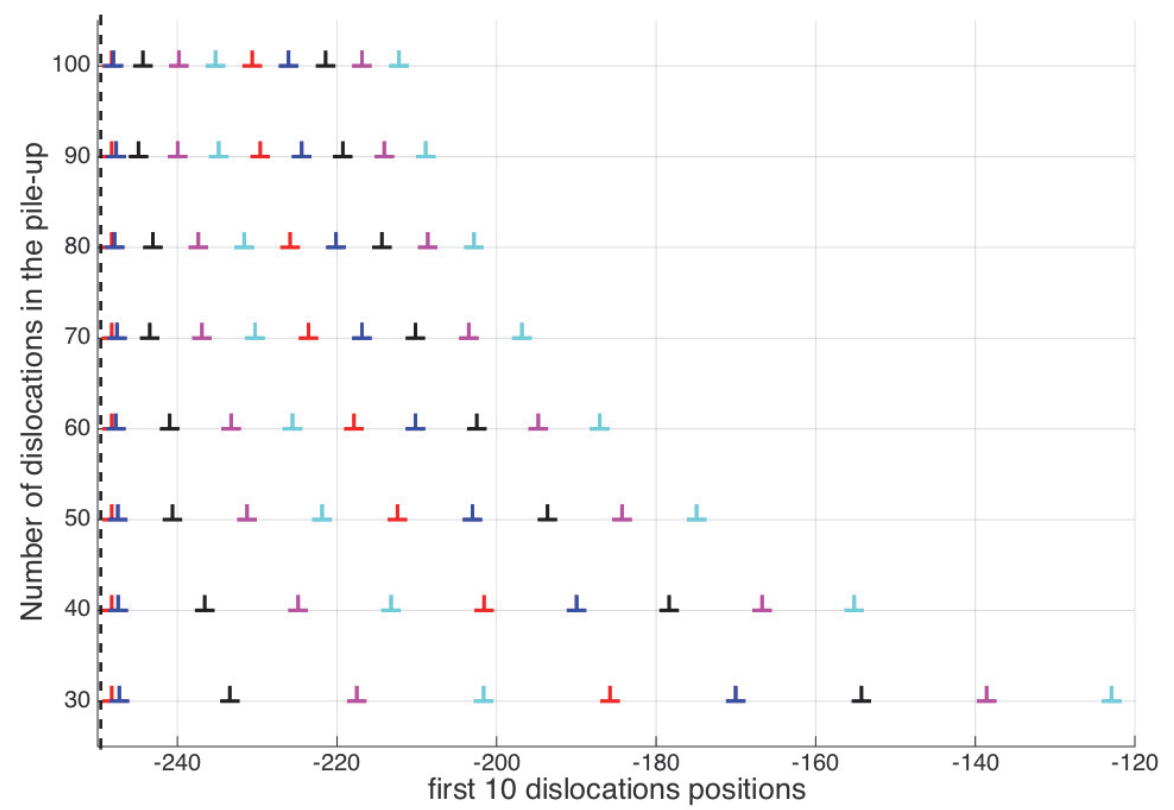

Figure 7: Variance of dislocation distribution near lock, depending on the total number of dislocations.

\subsubsection{Stacking fault energy affect on the pile-up length}

Up until now, we have been using $\eta$ that represents a homogeneous material. In general, $\eta$ can be fitted to ab initio calculations to represent more realistic stacking fault energy profiles (Gbemou et al. (2016)). Here we choose $\eta$ to have a local minimum energy to mimic the generalized stacking fault energy (GSFE). By using this technique, it is possible for a perfect dislocation to dissociate. Now we are interested in studying the effect of the stacking fault energy on the pile-up length at equilibrium. In a recently developed statistical pile-up model based on single-arm source (Pan et al. (2015)), the ratio of the length of the pile-up and the constant statistical value of the dislocation source length is studied as the primary parameter to measure pile-up effect in micropillars. Our results support their conclusion from a microscopic point of view since we take the behavior of each dislocation into consideration.

Fig. 8(a) shows the profile of the stacking fault energy $\eta$. Notice that now $\eta$ has two global minima at 
$\phi=0$ and $\phi=0.5$, but also has a local minimum at $\phi=0.25$. The local minimum represents the stacking fault energy, denoted by GSFE. We choose four different GSFE and solve the pile-up problem in Fig. 7 for 40 dislocations. It is observed in Fig. 8(b) that our model predicts a negative correlation between the stacking fault energy and the pile-up length. This is consistent with what has been found by the statistical model (Pan et al. (2015)).

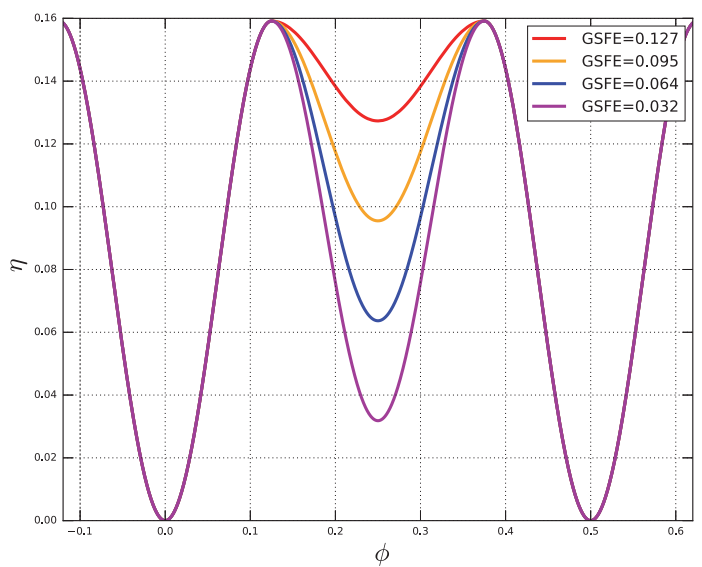

(a) $\eta$ profiles that have stacking fault effect

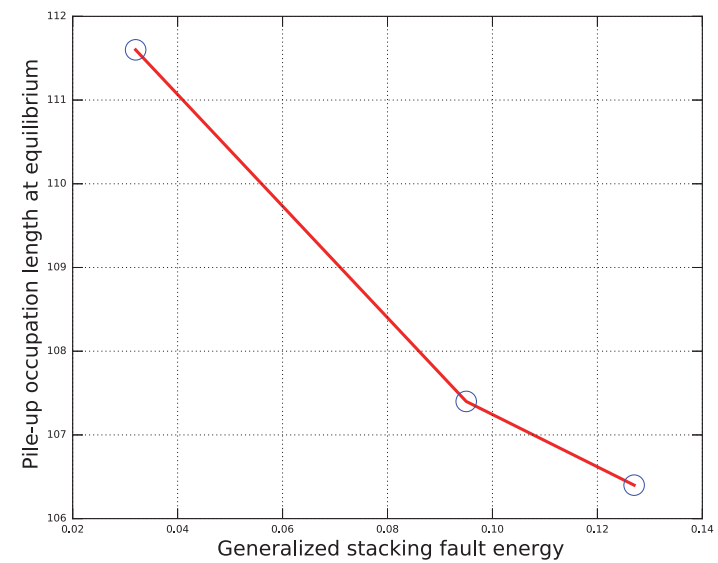

(b) Equilibrated pile-up occupation length corresponds to different $\eta$ profiles

Figure 8: Dislocation distribution near pile-up head depending on the total number of dislocations in the pile-up (from 30 to 100)

\subsection{Interlace: A group of positive dislocations attracted by a group of negative on a parallel adjacent slip plane}

Fujita (1958) and Head (1959) brought forward the problem of interlacing, where $n$ mobile positive edge dislocations are attracted by $n$ mobile negative dislocations on an adjacent parallel slip-plane. Fujita argues that there exists an equilibrated arrangement of such distributions called interlacing. Head solved this problem in details and postulates that a stable array could be formed when the dislocations are partially interlaced, i.e., a number of dislocations pairs would be formed but prevented from drifting apart by the attraction between the outer unpaired dislocations. The equilibrium is broken by applying sufficient shear load. This problem is investigated with our pile-up model. The geometry of Fig. 1 needs to be amended since we now need two parallel plastic layers. The domain is of the size $100 b \times 100 b$ for this case, with a spacing of $2 b$ between the two layers. The domain is uniformly discretized and refined to $h=0.2 b$ ( $h$ is the element dimension in $x$ ). Other simulation parameters are listed in Table 1.

This interlacing forming-breaking process is modeled. Without applying any load, the leading dislocations of adjacent planes attract each other until they get to the red equilibrated configuration, where an interlaced lock is formed between the first three pairs of dislocations. The dislocation core shapes of the interlaced 
configuration are shown in Fig. 9(a). The corresponding stress pattern near the interlacing region is shown in Fig. 9(b). At this moment, we apply a large enough shear load to break the interlacing lock, which leads to the formation of double pile-ups (colored in green).

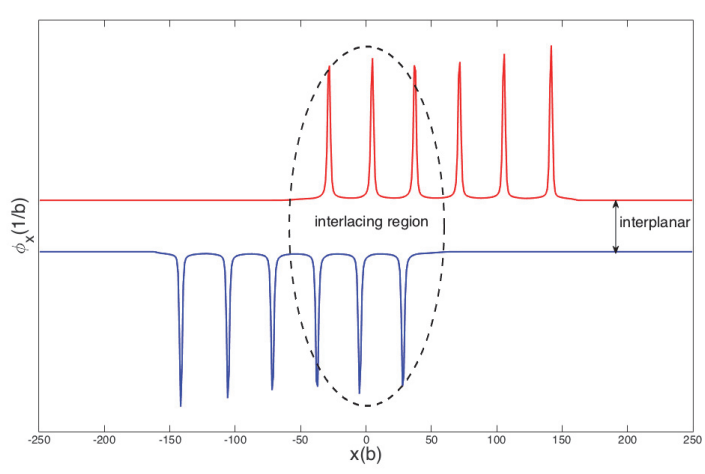

(a) interlacing core structures

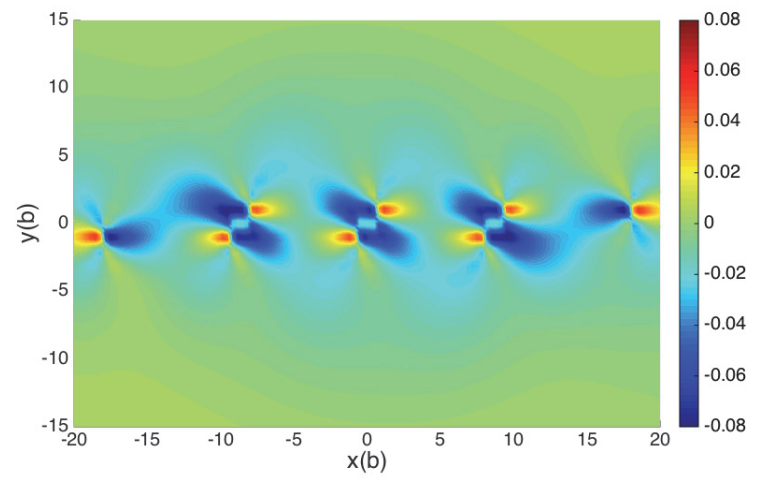

(b) $\sigma_{12}$ pattern close-up

Figure 9: Dislocation core shapes of the interlacing dislocation arrays; the circled 3 leading pairs form the interlacing lock that stablizes the system. Stress pattern close-up at the interlacing pairs.

Fig. 10 shows the stress associated with the interlacing process. A shear load $\tau^{a}=0.065 \mu$ is applied to the configuration of Fig. 10(b) to break the "interlacing bonds". The dislocations completely pass each other and form double pile ups (the end locks are manually put in by setting the dislocation velocity to zero), causing stress concentration on the boundaries. Note that the dislocations on the lower plane behave symmetrically with respect to those on the top.

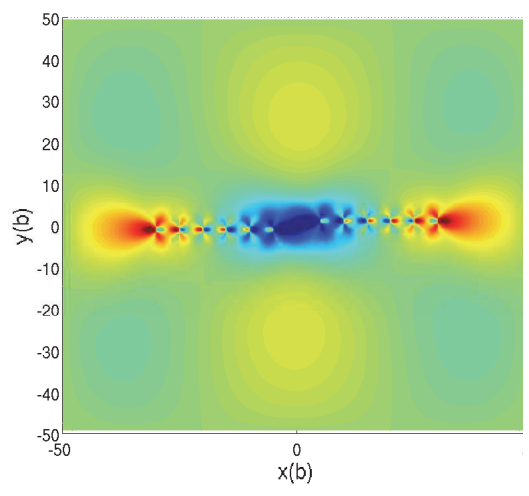

(a) $\sigma_{12}$ of initial configuration

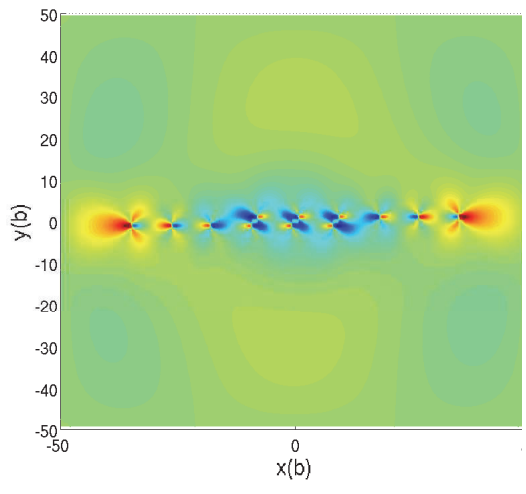

(b) $\sigma_{12}$ of interlacing equilibrium.

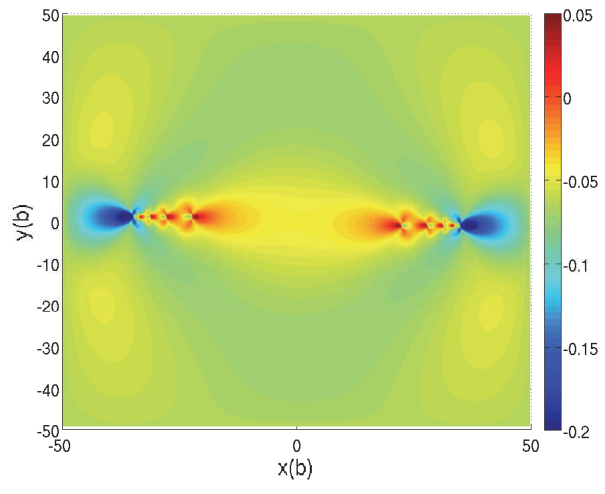

(c) $\sigma_{12}$ of double pile-ups

Figure 10: Interlaced dislocations on parallel adjacent slip planes form due to attraction. The lock is then broken by applying a shear load $\tau^{a}=0.065 \mu$ Under an externally applied shear load and form double pile-up eventually.

It is also interesting to point out that $\left\|\phi_{s}\right\|_{\infty}$ in this example differs from that of Fig. 3. $\left\|\phi_{s}\right\|_{\infty}$ in the interlacing simulations still asymptotically goes to zero but oscillates rapidly due to the much more complex driving force change in the system during the interlacing process. 


\subsection{A group of dislocations piles up against the interface of a bi-metallic body}

Consider the problem of a group of dislocations piles up against the interface of a bi-metallic body. The interface could bond either two isotropic or anisotropic materials with very different material properties. One of the interesting questions raised is how the stress is distributed in the dislocation-free body, which is of engineering importance for studying precipitation strengthening mechanism, mechanical stability, and interfacial cohesion with the matrix. This problem was originally proposed and analytically solved by Head (1953) using potential theory. The same problem was later studied by Chou (1966) who turned the problem into a singular integral equation of Cauchy type. Barnett (1967) extended the method to involve different Poisson's ratios and shear moduli between the two phases, leading to a complicated integral equation. Kuang and Mura (1968) considered edge dislocations that pile-up against a bimetallic interface using the same method. On the other hand, Roy et al. (2008) showed that neglecting the discrete effect leads to inaccurate results. Voskoboinikov et al. (2007b) resolves this issue by discretely modeling dislocations near the lock and matching the stress field with that of the far field, considering only isotropic materials. Those issues are naturally taken care of with our methodology: the short-range interactions are automatically sorted out via microscopically accurate kinematics. The stress field near a bi-material interface is shown in Fig. 11.

With our method, we are able to model the discrete behavior near the bimetallic interface. Fig. 12 shows the relationship between different applied shear stresses and the average spacing near the interface. (The average spacing is defined as the average spacing of the first three dislocations). We notice that the curve tends to flatten out with increasing load due to the fact that the repulsive forces in between the leading dislocations start to balance out the shear load and keep them from moving closer. Such an effect arises only when the discreteness is well captured. The inset plot shows the near interface dislocation behaviors, where we see that the cores of the two dislocations at the head of the pile-up have begun to overlap.

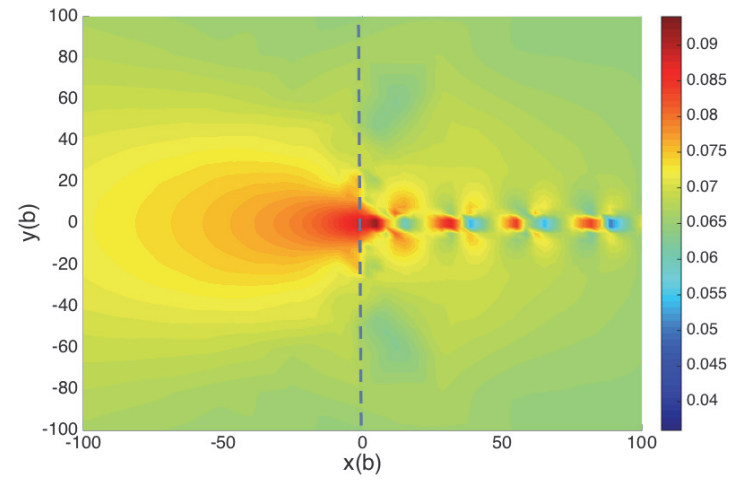

Figure 11: $\sigma_{12}$ pattern near interface-isotropic. Vertical dashed line denotes bimaterial interface.

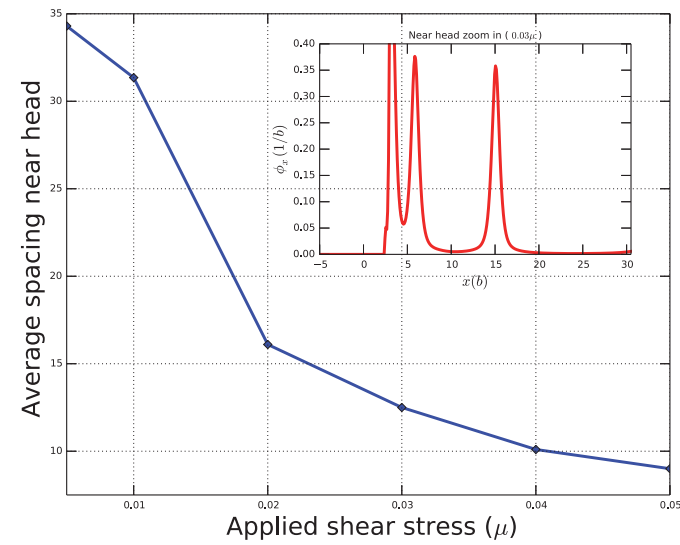

Figure 12: stress-spacing relations.

It is of engineering interest to understand how the bi-material difference affects the stress distribution of 
the dislocation-free body. For clarity, we denote the shear modulus and Poisson ratio of the left domain as $\mu_{1}$ and $\nu_{1}$; and that of the right as $\mu_{2}$ and $\nu_{2} \cdot \mu_{2}$ and $\nu_{2}$ are taken from Table 1 and held fixed. The interface of the bimetallic body is set to be impenetrable. A shear load is applied such that all dislocations of the right domain are forced to pile-up against the interface. A parametric study of 1) $\nu_{1} / \nu_{2}$ and 2) $\mu_{1} / \mu_{2}$ on the stress divergence and magnitude is performed below as shown in Fig. 13(a) and 13(b). The conclusion is that an increase in $\nu_{1}$ or $\mu_{1}$ over the neighboring $\nu_{2}$ and $\mu_{2}$ makes shear stress diverges slowly from the origin $(x=0)$ and thus lead to a higher stress on the dislocation-free side.

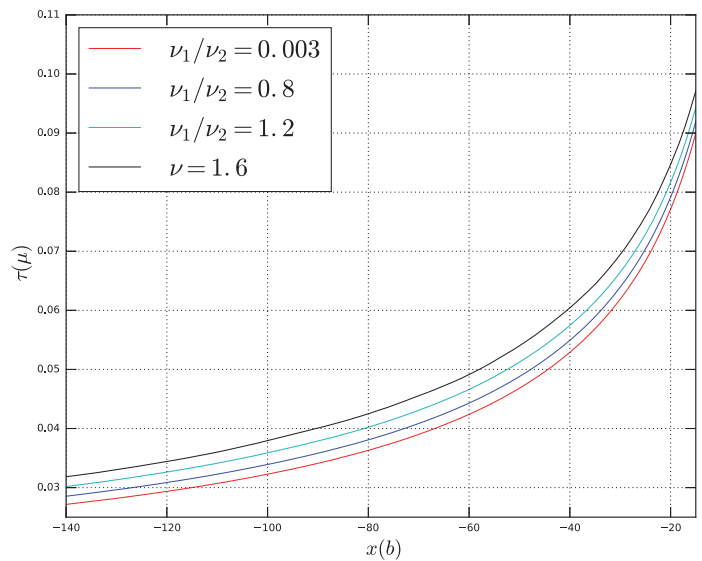

(a) trend of stress divergence for different $\nu_{1} / \nu_{2}$.

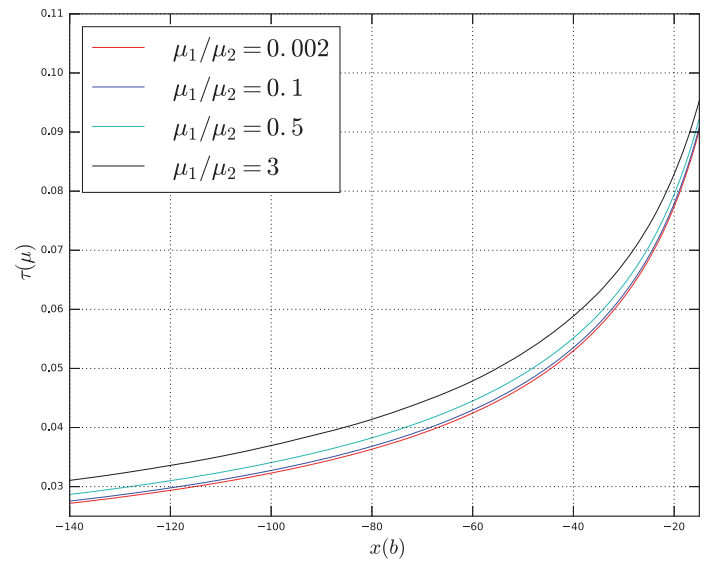

(b) trend of stress divergence for different $\mu_{1} / \mu_{2}$.

Figure 13: Parametric study of stress divergence trend.

To quantify the stress intensity, we fit the numerical data with a reciprocal function of the form

$$
\tau=\frac{A_{m}}{x^{\beta}+B_{m}},
$$

where $A_{m}$ is defined as the stress intensity factor, following Liu et al. (2014). Matlab cftool tool box is used for the fitting, which gives $\beta=0.01$. $A_{m}$ and $B_{m}$ are fitted accordingly. Notice that $\beta=1$ in Eq. 17 . Fig. 14 shows the different trends between the intensity factor and the $\mu$ and $\nu$ ratios. Notice that there exists an intersecting point at $A_{m} \approx 0.41$. For stress intensity $A_{m}<0.41$, the ratio of $\mu_{1} / \mu_{2}$ has a dominating effect on the stress intensity factor, while the ratio of $\nu_{1} / \nu_{2}$ is more affected when $A_{m}>0.41$. 


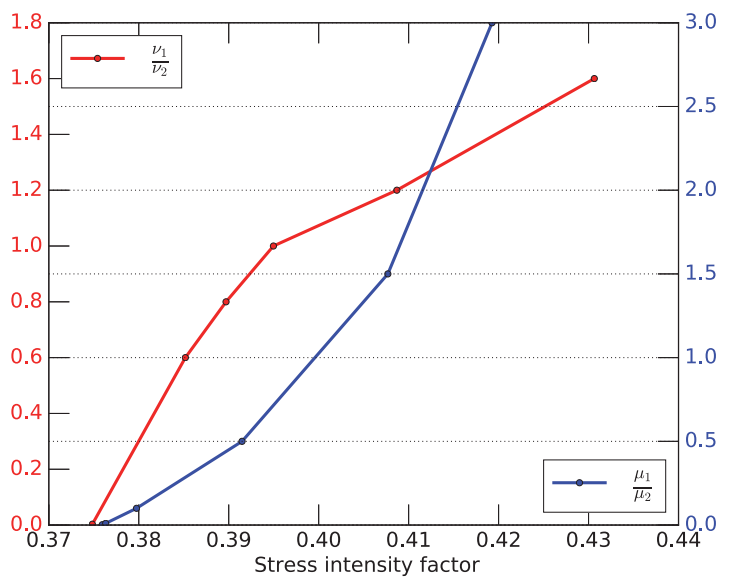

Figure 14: Stress intensity corresponding to different material strengths of bi-materials.

\subsubsection{Bimetallic interface in anisotropic materials}

Next we consider an anisotropic FCC crystal. As illustrated by Fig. 15, we take a typical FCC slip system (111)[101], and transform the coordinates to the global reference system $[\overline{1} 01] \times[1 \overline{2} 1] \times[111]$, such that the slip plane normal aligns with $y$ axis and slip direction with the $x$-axis. A shear load of magnitude $\tau^{a}=0.065 \mu$ is applied to force an array of dislocations to pile up against the bimetallic interface.

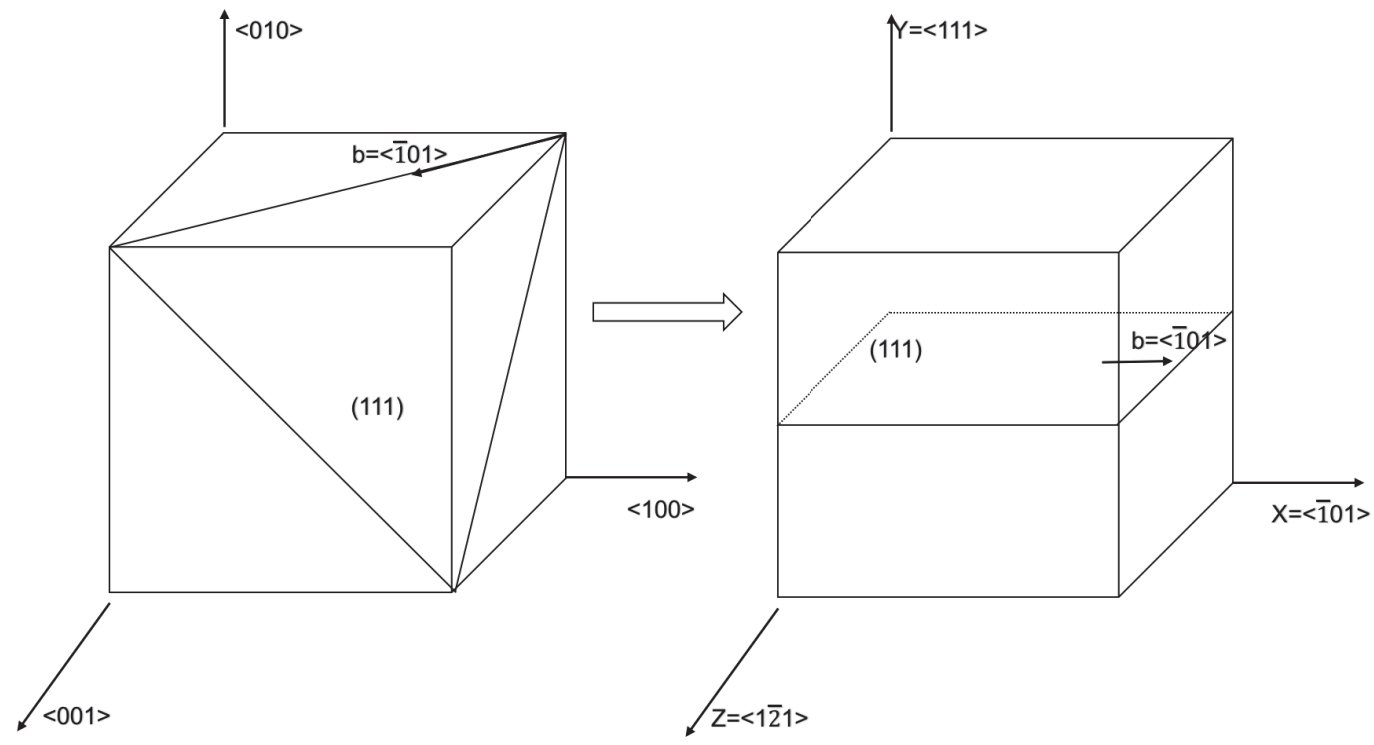

Figure 15: Coordinate transform illustration.

The anisotropic material properties are set to $C_{12}=12.5 G P a, C_{11}=14.8 G P a, C_{44}=10.8 G P a$ on the right (corresponding to Aluminum) and $C_{12}=61.9 \mathrm{GPa}, C_{11}=114.3 \mathrm{GPa}, C_{44}=31.6 \mathrm{GPa}$ on the left (corresponding to Copper). Fig. 16(a) shows the equilibrated shear stress field, which exhibits a different stress pattern than that from isotropic materials, and the equilibrated microstructure near the bi-metallic 
interface. The same form of function (18) is used to fit the stress divergence. For the chosen parameters, the isotropic case exhibits a slightly larger $A_{m}$ as shown in Fig. 16(b). However, a solid conclusion on the effect of anisotropy on stress intensity requires a thorough parametric study.

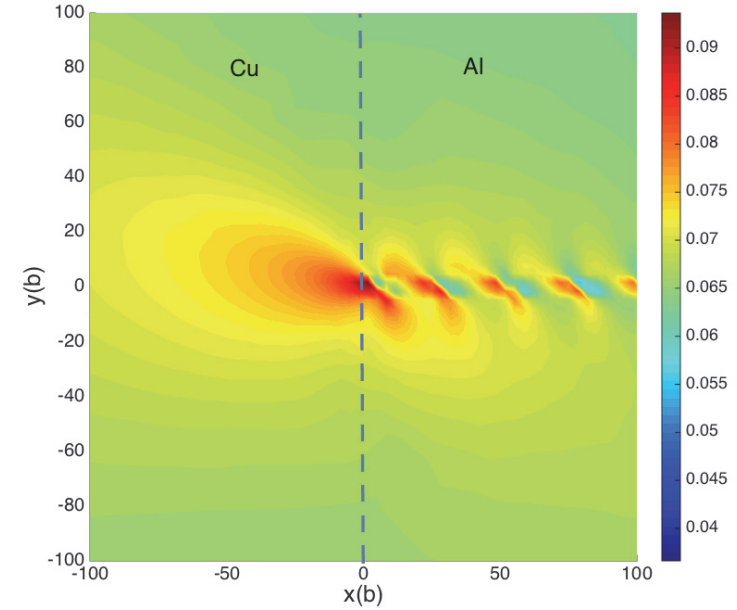

(a) $\sigma_{12}$ pattern near interface-anisotropic.

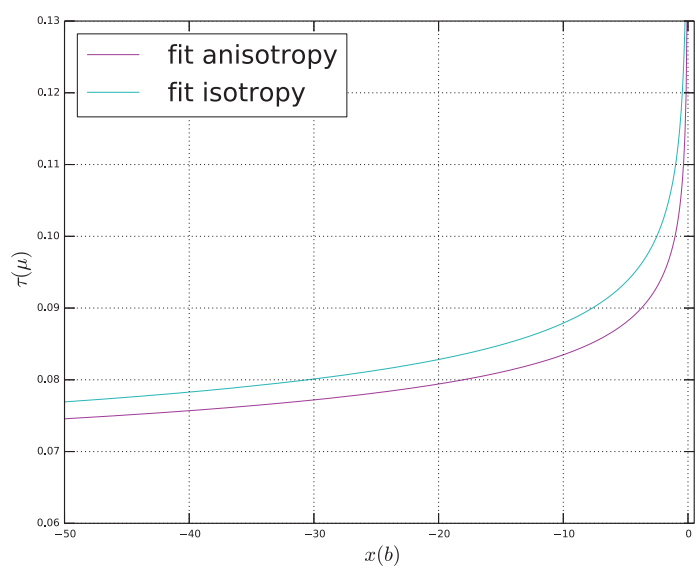

(b) Fit data with power-reciprocal functions, compared to isotropic case.

Figure 16: Dislocations pile up against anisotropic bimetallic interface. Vertical dashed line denotes bimaterial interface.

\subsection{Stress strain response of a single crystal body with randomly distributed edge dislocations}

Our model allows more general initial conditions, as dislocations are in reality seldomly regularly distributed. Fig. 17(a) shows a number of dislocations randomly initialized along five parallel slip planes. The slip planes have equal spacings of $180 b$ in the $y$-direction and the Finite Element mesh is locally refined around those layer areas. Fig. 17(b) shows the stress strain curve obtained from the body when subjected to a Dirichlet shear load. The shear stress, normalized by shear modulus, is calculated as the summation of reaction forces on the top face divided by the face area. The plastic flow associated with the motions of the randomly disributed dislocations, especially the annihilations of dislocations of opposite signs, gives rise to the nonlinear stress-strain response. Such short range interactions are often neglected in other continuum theories. In the pileup case, the mechanism that leads to hardening effect can be described as following: as the load increases and dislocations evolve and form pile-ups, the average spacing between dislocations decreases, which put up resistance to further dislocation motions. For random distributed dislocations of two signs, this process is more complicated because dislocations can annihilate during the evolution and that explains why the corresponding stress strain curve has more oscillations and also a smaller hardening rate. 


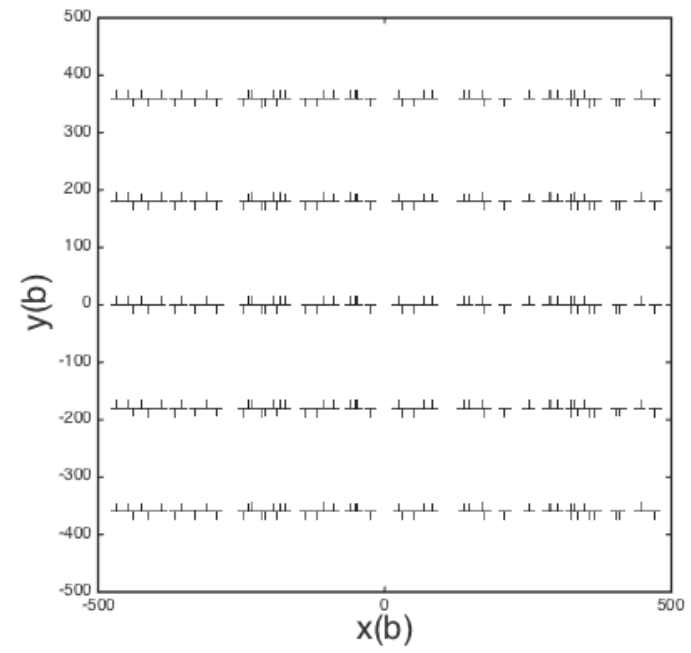

(a) random dislocations across 5 layers.

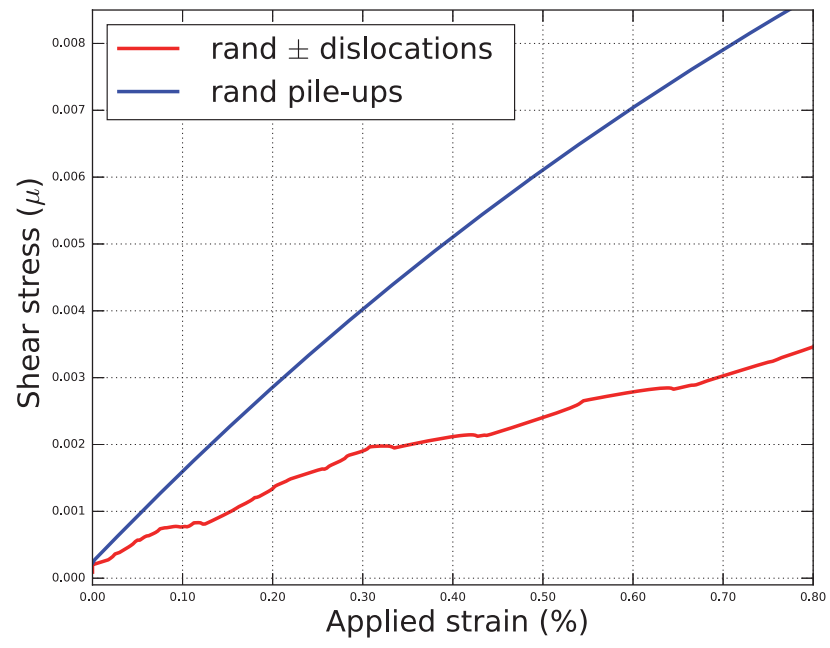

(b) stress strain curve

Figure 17: Stress strain curve for randomly distributed dislocations.

\section{Conclusions}

A 2-d dislocation pile-up model derived from the 3-d framework of Field Dislocation Mechanics is developed. The numerical examples presented in this work demonstrate the following characteristics of the new model: 1) we produce results consistent with analytical solutions; 2) problems that involving complex dislocation interactions such as dislocation coalescing and interlacing are well resolved; 3) dislocations are represented with a Nye tensor field so that the dislocation structures can be evolved by solving a timedependent partial differential equation of Hamilton-Jacobian form, and that the computational expense does not explicitly depend on the number of dislocations; 4) the dislocation velocity is constitutively chosen out of thermodynamical considerations such that the dissipation is guaranteed to be positive. A more accurate constitutive assumption can be achieved by fitting e.g., drag coefficients and stacking fault energy from finer scale simulations; Finally, since the model is essentially derived within the continuum mechanics context, anisotropy (induced from realistic FCC slip systems), inertia under high strain rate loading (such as shock impact) are naturally accounted for.

\section{Acknowledgement}

The author gratefully acknowledges Prof. Amit Acharya for many useful suggestions on the simulations. The author thanks Dr. Bertin and Dr. R. B. Sills for their help in editing the draft.

\section{References}

A. Acharya, A model of crystal plasticity based on the theory of continuously distributed dislocations, Journal of the Mechanics and Physics of Solids 49 (2001) 761-784. 
A. Acharya, Driving forces and boundary conditions in continuum dislocation mechanics, Proceedings of the Royal Society of London. Series A: Mathematical, Physical and Engineering Sciences 459 (2003) $1343-1363$.

A. Acharya, Constitutive analysis of finite deformation field dislocation mechanics, Journal of the Mechanics and Physics of Solids 52 (2004) 301-316.

A. Acharya, A. Roy, Size effects and idealized dislocation microstructure at small scales: predictions of a phenomenological model of mesoscopic field dislocation mechanics: Part I, Journal of the Mechanics and Physics of Solids 54 (2006) 1687-1710.

A. Acharya, New inroads in an old subject: plasticity, from around the atomic to the macroscopic scale, Journal of the Mechanics and Physics of Solids 58 (2010) 766-778.

A. Acharya, Microcanonical entropy and mesoscale dislocation mechanics and plasticity, Journal of Elasticity 104 (2011) 23-44.

A. Roy, A. Acharya, Finite element approximation of field dislocation mechanics, Journal of the Mechanics and Physics of Solids 53 (2005) 143-170.

S. Puri, A. Roy, A. Acharya, D. Dimiduk, Modeling dislocation sources and size effects at initial yield in continuum plasticity, Journal of Mechanics of Materials and Structures 4 (2010) 1603-1618.

S. Puri, A. Das, A. Acharya, Mechanical response of multicrystalline thin films in mesoscale field dislocation mechanics, Journal of the Mechanics and Physics of Solids 59 (2011a) 2400-2417.

S. Puri, A. Acharya, A. D. Rollett, Controlling plastic flow across grain boundaries in a continuum model, Metallurgical and Materials Transactions A 42 (2011b) 669-675.

V. Berdichevsky, D. Dimiduk, On failure of continuum plasticity theories on small scales, Scripta materialia $52(2005)$ 1017-1019.

S. D. Mesarovic, S. Forest, J. P. Jaric, Size-dependent energy in crystal plasticity and continuum dislocation models, in: Proc. R. Soc. A, volume 471, The Royal Society, 2015, p. 20140868.

J. Eshelby, F. Frank, F. Nabarro, The equilibrium of linear arrays of dislocations., Philosophical Magazine 42 (1951) 351-364.

E. Pacheco, T. Mura, Interaction between a screw dislocation and a bimetallic interface, Journal of the Mechanics and Physics of Solids 17 (1969) 163-170.

A. Head, The positions of dislocations in arrays, Philosophical Magazine 4 (1959) 295-302.

S. Akarapu, J. Hirth, Dislocation pile-ups in stress gradients revisited, Acta Materialia 61 (2013) 3621-3629. 
J. Ockendon, R. Voskoboinikov, S. Chapman, Continuum and discrete models of dislocation pile-ups. i pile-up at a lock, Journal of the Mechanics and Physics of Solids 55 (2007).

A. Roy, R. Peerlings, M. Geers, Y. Kasyanyuk, Continuum modeling of dislocation interactions: Why discreteness matters?, Materials Science and Engineering: A 486 (2008) 653-661.

C. L. Hall, Asymptotic analysis of a pile-up of edge dislocation, Materials Science and Engineering A (2011).

R. Voskoboinikov, S. Chapman, J. Ockendon, D. Allwright, Continuum and discrete models of dislocation pile-ups. i. pile-up at a lock, Journal of the Mechanics and Physics of Solids 55 (2007a).

R. Voskoboinikov, S. Chapman, J. Ockendon, Continuum and discrete models of dislocation pile-ups. ii. pileup of screw dislocations at the interface in a bimetallic solid, Philosophical magazine letters 87 (2007b) 669-676.

R. Voskoboinikov, S. Chapman, J. Mcleod, J. Ockendon, Asymptotics of edge dislocation pile-up against a bimetallic interface, Mathematics and Mechanics of Solids 14 (2009) 284-295.

P. van Meurs, A. Muntean, Upscaling of the dynamics of dislocation walls, arXiv preprint arXiv:1411.0870 (2014).

L. Scardia, R. H. Peerlings, M. A. Peletier, M. G. Geers, Mechanics of dislocation pile-ups: a unification of scaling regimes, Journal of the Mechanics and Physics of Solids 70 (2014) 42-61.

J. Rezaei Mianroodi, R. Peerlings, B. Svendsen, Strongly non-local modelling of dislocation transport and pile-up, Philosophical Magazine 96 (2016) 1171-1187.

A. Acharya, X. Zhang, From dislocation motion to an additive velocity gradient decomposition, and some simple models of dislocation dynamics (2014).

X. Zhang, A. Acharya, N. J. Walkington, J. Bielak, A single theory for some quasi-static, supersonic, atomic, and tectonic scale applications of dislocations, Journal of the Mechanics and Physics of Solids 84 (2015) $145-195$.

C. Shen, Y. Wang, Phase field model of dislocation networks, Acta materialia 51 (2003) 2595-2610.

Y. U. Wang, Y. Jin, A. Cuitino, A. Khachaturyan, Nanoscale phase field microelasticity theory of dislocations: model and 3d simulations, Acta Materialia 49 (2001) 1847-1857.

Y. Wang, J. Li, Phase field modeling of defects and deformation, Acta Materialia 58 (2010) 1212-1235.

S. Hu, Y. Li, Y. Zheng, L. Chen, Effect of solutes on dislocation motiona phase-field simulation, International Journal of Plasticity 20 (2004) 403-425. 
Y. Xiang, H. Wei, P. Ming, E. Weinan, A generalized peierls-nabarro model for curved dislocations and core structures of dislocation loops in al and cu, Acta materialia 56 (2008) 1447-1460.

J. Mianroodi, A. Hunter, I. J. Beyerlein, B. Svendsen, Theoretical and computational comparison of models for dislocation dissociation and stacking fault/core formation in fcc crystals, Journal of the Mechanics and Physics of Solids 95 (2016) 719-741.

B. Pan, Y. Shibutani, X. Zhang, F. Shang, Effect of dislocation pile-up on size-dependent yield strength in finite single-crystal micro-samples, Journal of Applied Physics 118 (2015) 014305.

J. R. Rice, Inelastic constitutive relations for solids: an internal-variable theory and its application to metal plasticity, Journal of the Mechanics and Physics of Solids 19 (1971) 433-455.

A. Das, A. Acharya, J. Zimmer, K. Matthies, Can equations of equilibrium predict all physical equilibria? a case study from field dislocation mechanics, Mathematics and Mechanics of Solids (2012).

L. P. Kubin, G. Canova, M. Condat, B. Devincre, V. Pontikis, Y. Bréchet, Dislocation microstructures and plastic flow: a 3d simulation, Solid State Phenomena 23 (1992) 455-472.

S. S. Chakravarthy, W. Curtin, Effect of source and obstacle strengths on yield stress: A discrete dislocation study, Journal of the Mechanics and Physics of Solids 58 (2010) 625-635.

K. Gbemou, V. Taupin, J. Raulot, C. Fressengeas, Building compact dislocation cores in an elasto-plastic model of dislocation fields, International Journal of Plasticity 82 (2016) 241-259.

F. Fujita, Dislocation theory of fracture of crystals, Acta metallurgica 6 (1958) 543-551.

A. Head, Edge dislocations in inhomogeneous media, Proceedings of the Physical Society. Section B 66 (1953) 793.

Y. Chou, Equilibrium of linear dislocation arrays in heterogeneous materials, Journal of Applied Physics 37 (1966) 2425-2429.

D. Barnett, The effect of shear modulus on the stress distribution produced by a planar array of screw dislocations near a bi-metallic interface, Acta Metallurgica 15 (1967) 589-594.

J.-G. Kuang, T. Mura, Dislocation pile-up in two-phase materials, Journal of Applied Physics 39 (1968) 440 $\quad 109-119$.

D. Liu, Y. He, B. Zhang, L. Shen, A continuum theory of stress gradient plasticity based on the dislocation pile-up model, Acta Materialia 80 (2014) 350-364. 


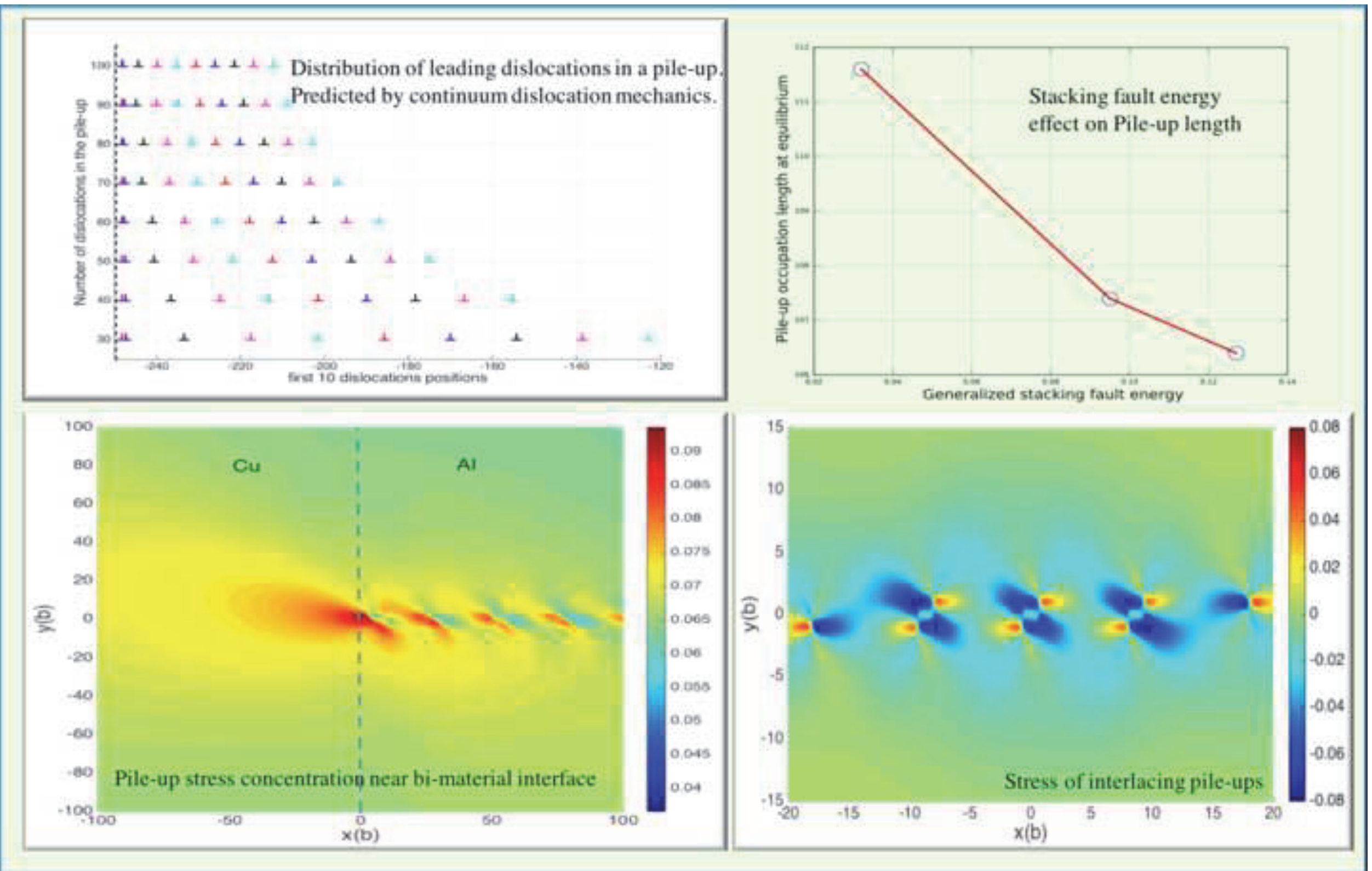

